\title{
Robust Speed Limits Scheme Design for Bimodal Transportation Systems
}

\author{
Ke Yu, ${ }^{1}$ Yanni Yang, ${ }^{1,2}$ and Lang Fan ${ }^{2}$ \\ ${ }^{1}$ Institute of Transportation Engineering, Tsinghua University, Beijing 100084, China \\ ${ }^{2}$ Information School, Capital University of Economics and Business, Beijing 100070, China \\ Correspondence should be addressed to Yanni Yang; yanrui4lanyu@126.com
}

Received 20 April 2014; Accepted 2 July 2014; Published 3 August 2014

Academic Editor: Evangelos J. Sapountzakis

Copyright (c) $2014 \mathrm{Ke} \mathrm{Yu} \mathrm{et} \mathrm{al.} \mathrm{This} \mathrm{is} \mathrm{an} \mathrm{open} \mathrm{access} \mathrm{article} \mathrm{distributed} \mathrm{under} \mathrm{the} \mathrm{Creative} \mathrm{Commons} \mathrm{Attribution} \mathrm{License,} \mathrm{which}$ permits unrestricted use, distribution, and reproduction in any medium, provided the original work is properly cited.

\begin{abstract}
In the context of travel demand uncertainty, this paper investigates how to determine the robust road speed limits for improving mobility and lowering vehicular emissions in bimodal transportation systems that involve private cars and subway. More specifically, the total demand vector is supposed to vary within a given set. Our target is to find the optimal road speed limits against the worst feasible demand scenario so as to minimize the sum social cost of system travel time and vehicular emissions. In order to estimate traffic emissions more reliably, motor vehicle emission simulator (MOVES) is utilized to simulate the emission factor function with respect to average speed. On these bases, we formulate the robust speed limits design problem as a "min-max" nonlinear model with complementarity constraints and solve it iteratively by a cutting-plane scheme that contains two sub-MPCCs. A numerical example is illustrated at the end.
\end{abstract}

\section{Introduction}

As the ownership of motor vehicles is constantly increasing, traffic congestion alleviation has been the paramount mission for traffic management agency. Recently, traffic emissions attract more attention because of worsening of the air quality. The hazy weather occurs frequently in Beijing since the end of 2012. It is reported that excessive concentration of $\mathrm{PM}_{2.5}$ (particulate matter smaller than 2.5 micrometers) in the atmosphere is the prime cause of hazy weather, and the $\mathrm{PM}_{2.5}$ emissions from mobile sources account for $25 \%$. In addition, longtime exposure to $\mathrm{PM}_{2.5}$ would cause respiratory infections, asthma, lung cancer, and other diseases. Therefore, traffic pollution harms human health and it should be addressed together with traffic congestion for traffic management.

Scholars investigate some countermeasures, that is, road pricing, road space rationing, imposing speed limits, and so forth, to mitigate traffic congestion and reduce vehicular emissions. Moreover, most previous studies are concentrated on road pricing (see, e.g., [1-3], for recent reviews) that is theoretically advocated as an effective instrument to internalize traffic negative externalities since the pioneering work by Pigou [4]. However, the public acceptance of road pricing is quite poor that restricts it to be implemented in practice [1]. In contrast, it is easier and more widespread to impose speed limits in practical traffic management, which is the concern of this paper.

Setting speed limits is widely used to reduce traffic crashes, because a higher driving speed contributes to a higher fatality risk. The work of Joksch [5] concluded that taking the fatality risk involved in a traffic crash at $40 \mathrm{mph}$ as a reference, the fatality risk rises 2.5 -fold at $60 \mathrm{mph}, 6$ fold at $70 \mathrm{mph}$, and approximately 20 -fold at $80 \mathrm{mph}$. Besides, imposing speed limits also serves the purpose of reducing traffic emissions and fuel consumption [6]. Texas Transportation Commission created environmental speed limits to lower vehicular emissions and meet federal air quality standards at the request of Texas Commission on Environmental Quality (TCEQ) [7]. Madireddy et al. [8] showed that $\mathrm{CO}_{2}$ and $\mathrm{NO}_{x}$ emissions were reduced by approximately $25 \%$ in residential areas when speed limits declined from 50 to $30 \mathrm{~km} / \mathrm{h}$. Yang et al. $[9,10]$ examined how speed limits affect the network-wide pollutant emissions and revealed 
that a suitable speed limits setting has the potential of reducing traffic emissions and accidents. In addition to safety and environment considerations, imposing speed limits is capable of altering traffic flow distribution at equilibrium and further influencing the system performance. Woolley et al. [11] and Madireddy et al. [8] employed microscopic traffic simulation tools to investigate the influence of speed limit setting on network system performance and suggested that speed limits can reallocate traffic flow distribution. Yang et al. [12] demonstrated that imposing speed limits can decentralize the traffic flow pattern just as road pricing from macroscopic network equilibrium perspective. Wang [13] investigated the impact of setting speed limits, on network efficiency and equity, and developed a bilevel programming model to find the optimal speed limit scheme that maximizes the network efficiency. Yang et al. $[9,10]$ envisioned a variable speed limits system to achieve better efficiency, safety, and environment sustainability on traffic networks. Since that vehicular emissions not only are nonmonotonic functions of vehicular speeds [14] but also are associated with many other factors such as vehicle acceleration, fuel type, and local weather conditions [15], Yang et al. [9, 10] employed MOVES to calculate traffic emissions.

To our best knowledge, these existing studies on speed limits design only considered road networks and made the hypothesis that the traffic demand is fixed. Nevertheless, an urban transportation system usually involves multiple transport modes and the total travel demand is uncertain. To fill this gap, this paper attempts to explore the speed limits design problem under demand uncertainty in bimodal transportation systems. First of all, we propose a synthesized link performance function and establish user equilibrium model for our bimodal networks. Given a speed limit scheme, the UE flow pattern is determined by the proposed model. To achieve both minimum system travel time and minimum traffic emissions with demand uncertainty, we then assume that the total demand belongs to a given set and develop a "min-max" model to find the optimal road speed limits against the worst-case feasible demand scenario for bimodal networks. Furthermore, emission simulator (MOVES) is utilized to simulate emission factor functions to make the emission estimation more reliable, and a cutting plane is applied to solve the robust speed limits design model.

For the remainder, Section 2 describes the specific bimodal network structure composed of two modes and proposes the link performance function in such a combined system. Subsequently, the user equilibrium model for a bimodal network under speed limits is developed. Section 3 makes use of emission simulator (MOVES) to obtain emission factor functions and proposes a robust speed limits design model formulated as a "min-max" nonlinear problem with complementarity constraints to minimize the sum social cost of system travel time and emissions. To describe the demand uncertainty, the total demand is assumed to vary within a given set. Section 4 conducts a cutting plane scheme to solve the proposed model and applies the manifold suboptimization algorithm to solve two sub-MPCCs. Section 5 illustrates how to design the optimal robust speed limits with a numerical example and conducts the sensitive analysis of parking fees. Finally, this paper is ended with conclusions.

\section{UE with Speed Limits in Bimodal Networks}

Multimodal network equilibrium problem has been well studied in the literature (e.g., [16-19]). Ying and Yang [20] proposed a stochastic user equilibrium model for congested bimodal networks and optimized road pricing with sensitive analysis method. Hamdouch et al. [21] extended the pricing design framework to multimodal transportation networks. Wu et al. [22] developed a multimodal network equilibrium model and designed a Pareto-improving pricing scheme to reduce congestion for multimodal transportation networks. $\mathrm{Fu}$ and Lam [19] formulated an activity-based network equilibrium model to schedule daily activity-travel patterns for multimodal networks. Slightly different from the previous research, we explore the bimodal network equilibriummodeling while considering speed limits. Two travel modes in our transportation system, private cars and subway, are considered in this paper and the following assumptions are made before modeling work.

(a) Travelers can only choose a preferring mode; their mode choice behaviors follow the principle of utility maximization and can be described by a bimodal Logit model.

(b) For each origin-destination (O-D) pair, the total demand is tentatively fixed in this section.

(c) The speed limits can be only imposed on road links.

(d) A 100\% compliance of the speed limits is ensured by autoenforcement system. In other words, all travelers utilizing road links drive completely inconsistent with the posted speed limits.

In our research, the combined bimodal network, denoted by $G(N, \Omega)$, involves two subnetworks: road network and subway network, where $N$ is the set of nodes and stations and $\Omega$ is the set of road links and subway segments. According to the link characteristics, $\Omega$ can be divided into road link set $\Omega^{r}$ and subway segment set $\Omega^{s}$. Henceforth, the superscripts $r$ and $s$ represent private car mode and subway mode, respectively. Moreover, subway segments are further classified into three categories: subway links, transfer links, and embarking or alighting links. Generally, a subway line is composed of serial subway links. Passengers can change subway lines by transfer links in transfer stations and must pass through embarking or alighting links between stations and centroids (origins or destinations). Figure 1 applies an example to demonstrate the basic structure of a bimodal network, which consists of an O-D pair (1-3), 6 nodes, and 9 links. Note that S1-S2-S3 and 2a-S3 are two separate subway lines. In fact, $\mathrm{S} 2$ and $2 \mathrm{a}$ are the same station. To specify the transfer link within station S2 more distinctly, we add a virtual station $2 \mathrm{a}$ in this network.

Let $W$ denote the set of origin-destination (O-D) pairs; let $M$ denote the set of transportation modes; that is, $M=\{r, s\}$. For each O-D pair $w \in W$ by mode $m \in M$, the travel demand 


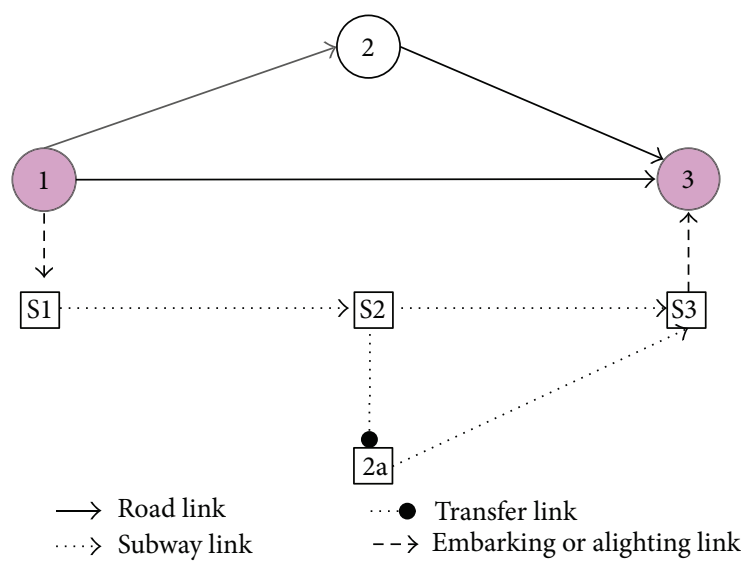

FIgURE 1: Basic structure of a bimodal network.

is denoted by $d_{m}^{w}$. Thus, the total travel demand between $\mathrm{O}-$ D pair $w$ with respect to all modes is represented by $q^{w}$, tentatively assumed to be a constant in this section. Let $x_{m}^{w}$ be the passenger link flow vector for O-D pair $w$ by mode $m$, and let $A$ be the node-link incidence matrix. To represent the flow balance constraints between O-D pair $w$, we define $E_{m}^{w}$ as its "input-output" vector, that is, a vector that has exactly two nonzero components: 1 and -1 , corresponding to the origin node and the destination node, respectively. For each link $a \in \Omega, v_{a, m}$ represents the traffic flow on link $a$ by mode $m$. The feasible region $\Lambda$ for the bimodal network equilibrium flow model can be given as follows:

$$
\begin{gathered}
\sum_{m \in M} d_{m}^{w}=q^{w}, \quad \forall w \in W, \\
A x_{m}^{w}=E_{m}^{w} d_{m}^{w}, \quad \forall w \in W, \quad \forall m \in M, \\
v_{a, m}=\sum_{w \in W} x_{a, m}^{w}, \quad \forall a \in \Omega, \quad \forall m \in M, \\
x_{a, m}^{w} \geq 0, \quad \forall w \in W, a \in \Omega, \quad \forall m \in M .
\end{gathered}
$$

Equation (1) expresses that for each O-D pair, the total demand is equivalent to the sum of travel demands by subway and private cars. Equation (2) is the flow balance constraint. Equation (3) defines the aggregate link flow and (4) ensures that the link flow by each mode is nonnegative. All the feasible $(v, d)$ are yielding to the above region $\Lambda$.

For each link $a \in \Omega^{r}$, we denote $s_{a}$ by the imposed speed limit. Let $\bar{t}_{a}$ represent the least travel time under the speed limit $s_{a}$ on link $a$; then we have $\bar{t}_{a}=l_{a} / s_{a}$, where $l_{a}$ is the length of road link $a$. Denote $v_{a}$ by the amount of traffic flow on link $a$. The original link travel time function $t_{a}\left(v_{a}\right)$ is assumed to be continuously increasing, differentiable, convex, and strictly monotone without considering speed limits. However, in presence of speed limits, the link performance function is expressed as $\widetilde{t}_{a}\left(v_{a}\right)$, and the equation $\widetilde{t}_{a}\left(v_{a}\right)=\max \left(t_{a}\left(v_{a}\right), \bar{t}_{a}\right)$ holds under the assumption of $100 \%$ speed limits' compliance [12]. $\widetilde{t}_{a}\left(v_{a}\right)$ is continuous, convex, and monotonically increasing, but not differentiable at $\bar{v}_{a}=$ $t_{a}^{-1}\left(\bar{t}_{a}\right)$ as shown in Figure 2.

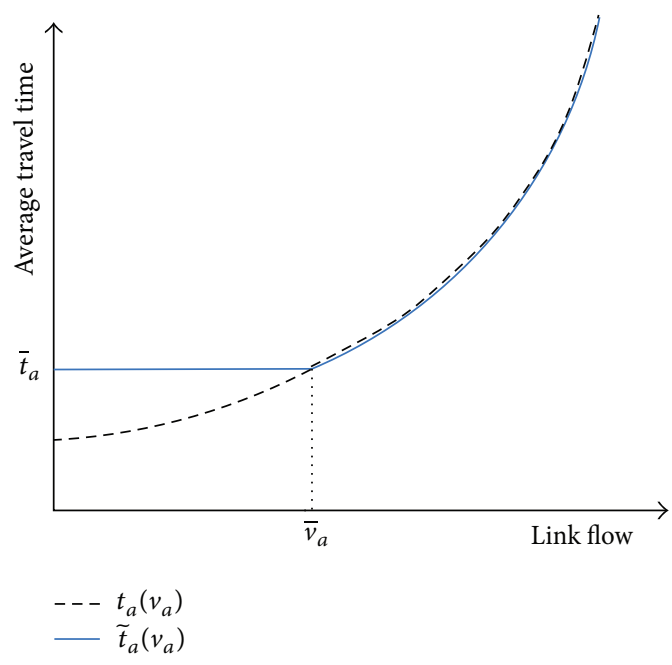

FIgURE 2: Travel time function under speed limit.

Yang et al. [12] showed that UE model under speed limits can be solved by existing traffic assignment methods, for example, Frank-Wolf method, and proved that the uniqueness of link travel time at user equilibrium (UE) still holds. For the links with nonbinding speed limits, the link flows at equilibrium are definitely unique. But for those links with binding speed limits, the UE flows are not necessarily unique.

Different from road network, subway segments are classified into three categories: subway links, transfer links, and embarking or alighting links. The travel time on subway link includes the running time TR and stop interval TS between two adjacent stations, both of which are fixed and independent of the amount of passengers. For each transfer link or embarking or alighting link, the travel time generally includes passengers walking time TP and waiting time TW. We suppose that walking time is associated with the amount of passengers and can be expressed as a nonlinear function of link passenger flow, that is, $\operatorname{TP}\left(v_{a}\right)=t_{a}^{0}\left(1+\alpha\left(v_{a} / C_{a}\right)^{\beta}\right)$, where $t_{a}^{0}$ is the passenger flow free walking time and $\alpha$ and $\beta$ are calibrated parameters. Assuming that passenger arrival rate is uniform, the waiting time for subway line $i$ is $0.5 / f_{i}$ [23], where $f_{i}$ is the frequency of line $i$. Note that the waiting time for alighting links is zero. In order to generalize the representation, the link performance function for subway network is given by

$$
\tilde{t}_{a}\left(v_{a}\right)= \begin{cases}\mathrm{TP}\left(v_{a}\right)+\mathrm{TW}_{a}, & \forall a \in \Omega^{s 1} \\ \mathrm{TR}_{a}+\mathrm{TS}_{a}, & \forall a \in \Omega^{s 2},\end{cases}
$$

where $\mathrm{TW}_{a}=\sum_{i} 0.5 / f_{i} \delta_{a i}$ and $\delta_{a i}$ is a $0-1$ variable. $\delta_{a i}=$ 1 when passengers can arrive at line $i$ through link $a$. Otherwise, $\delta_{a i}=0 . \Omega^{s 1}$ is denoted by the set of transfer, embarking, and alighting links. $\Omega^{s 2}$ is denoted by the set of subway links. We have $\Omega^{s 1} \cup \Omega^{s 2}=\Omega^{s}$. Thus, the synthesized 
link performance function for the bimodal network can be written as shown below:

$$
\begin{aligned}
& \tilde{t}_{a, m}\left(v_{a, m}, s_{a, m}\right) \\
& = \begin{cases}\max \left(t_{a, m}\left(v_{a, m}\right), \bar{t}_{a, m}\left(s_{a, m}\right)\right), & \forall a \in \Omega^{r}, \\
\operatorname{TP}_{a, m}\left(v_{a, m}\right)+\mathrm{TW}_{a, m}, & \forall a \in \Omega^{s 1}, \\
\operatorname{TR}_{a, m}+\mathrm{TS}_{a, m}, & \forall a \in \Omega^{s 2} .\end{cases}
\end{aligned}
$$

We make a hypothesis that, within the same transportation mode, travelers' behaviors are consistent with the Wardrop user equilibrium principle that all the utilized paths between an arbitrary O-D pair experience the same and least travel time and the travel time along unutilized paths is definitely equal to or longer than the former one. In this case, the following user equilibrium condition for each mode holds:

$$
\begin{gathered}
\widetilde{t}_{p, m}^{w} \begin{cases}=c_{m}^{w} & \text { if } h_{p, m}^{w} \geq 0, \\
\geq c_{m}^{w} & \text { if } h_{p, m}^{w}=0,\end{cases} \\
\forall w \in W, m \in M, p \in P_{m}^{w},
\end{gathered}
$$

where $P_{m}^{w}$ is the set of paths between O-D pair $w$ by mode $m, \widetilde{t}_{p, m}^{w}$ represents the travel time along path $p$ between $\mathrm{O}-\mathrm{D}$ pair $w$ by mode $m$, and $h_{p, m}^{w}$ is the corresponding path flow. $c_{m}^{w}$ implies the equilibrium travel time between O-D pair $w$ by mode $m$.

Moreover, travelers' modal choice is in the light of the generalized travel cost for each mode, which is assumed to include the equilibrium travel time and tolling in this paper and can be formulated as follows:

$$
\mu_{m}^{w}=\theta c_{m}^{w}+\tau_{m}^{w}, \quad \forall w \in W, m \in M,
$$

where $\mu_{m}^{w}$ denotes the generalized travel cost of mode $m$ between O-D pair $w$. $\theta$ can be interpreted as the value of time. $\tau_{m}^{w}$ represents the extra payment for travelers using mode $m$ between O-D pair $w$. More specifically, $\tau_{r}^{w}$ can be considered as parking fare at the destination. $\tau_{s}^{w}$ indicates the subway ticket fare and can be implemented in various manners. For example, the subway ticket fare is ride-based and fixed as $2 ¥$ in Beijing, while it is a link-based charge in Shanghai. It is assumed that travelers' mode choice behavior can be described by a multinomial Logit model as follows:

$$
d_{m}^{w}=q^{w} \frac{\exp \left(-\mu_{m}^{w}\right)}{\sum_{m \in M} \exp \left(-\mu_{m}^{w}\right)}, \quad \forall w \in W .
$$

The multimodal traffic network equilibrium conditions can be described by the following variational inequality (VI) model [24]. Consider the following:

$$
\widetilde{t}\left(v^{*}, s\right)^{T}\left(v-v^{*}\right)+\psi\left(d^{*}\right)^{T}\left(d-d^{*}\right) \geq 0, \quad \forall(v, d) \in \Lambda .
$$

At least one optimal solution $\left(v^{*}, d^{*}\right)$ must exist because the objective function is continuous and the feasible region is a compact polyhedron [25].
According to the Karush-Kuhn-Tucker (KKT) conditions associated with the above variational inequality problem, we obtain the following equivalent multimodal network equilibrium conditions:

$$
\begin{gathered}
x_{a, m}^{w}\left(\tilde{t}_{a, m}\left(v_{a, m}, s_{a, m}\right)-\pi_{i, m}^{w}+\pi_{j, m}^{w}\right)=0, \\
\forall a=(i, j) \in \Omega, w \in W, m \in M, \\
\tilde{t}_{a, m}\left(v_{a, m}, s_{a, m}\right)-\pi_{i, m}^{w}+\pi_{j, m}^{w} \geq 0, \\
\forall a=(i, j) \in \Omega, w \in W, m \in M, \\
d_{m}^{w}=q^{w} \frac{\exp \left(-\theta\left(\pi_{o, m}^{w}-\pi_{d, m}^{w}\right)-\tau_{m}^{w}\right)}{\sum_{m \in M} \exp \left(-\theta\left(\pi_{o, m}^{w}-\pi_{d, m}^{w}\right)-\tau_{m}^{w}\right)}, \\
\forall w \in W,
\end{gathered}
$$

$$
(v, d) \in \Lambda \text {, }
$$

where $\pi_{m}^{w}$ is the node potential vector $[26,27]$ for mode $m$ between O-D pair $w$.

The mathematical programming model for this bimodal network equilibrium problem can be formulated as follows:

$$
\begin{aligned}
\min Z= & \sum_{a \in \Omega} \sum_{m \in M} \int_{0}^{v_{a, m}} \tilde{t}_{a, m}\left(\omega, s_{a, m}\right) d \omega \\
& +\sum_{w \in W} \int_{0}^{d_{s}^{w}} \frac{1}{\theta}\left(\ln \frac{\sigma}{d^{w}-\sigma}+\tau_{s}^{w}-\tau_{r}^{w}\right) d \sigma
\end{aligned}
$$

s.t. $\quad(v, d) \in \Lambda$,

where $d_{s}^{w}$ is the traffic demand for subway mode. This model is a nonlinear problem with linear constraints and can be solved by many existing algorithms. Given a speed limit scheme, we can obtain the UE flow distribution and traffic demand for each mode by solving this model.

\section{Robust Speed Limits Design Model}

As aforementioned, this paper aims to find out a robust speed limits scheme on road network to minimize the total social cost for a bimodal transportation system, which is referred to as the summation of the social cost of system travel time and network-wide emissions. So how to calculate traffic emissions is the primary issue before developing robust speed limits design model.

3.1. Traffic Emissions Functions Extracted from MOVES. Usually, traffic emissions can be estimated by two different approaches: (1) making use of the existing traffic emission calculation models or software, such as CMEM and MOVES; (2) fitting traffic emissions functions through extracting samples from those available emission calculators [28]. The former one needs various input parameters, that is, weather and driving mode distribution, and is definitely more timeconsuming (US Environmental Protection Agency). Consequently, we can receive more accurate and reliable results, 
at a price of time cost. When this method is employed to calculate traffic emissions in a network optimization problem, it requires conducting a significant number of calculations to obtain the optimal solution. More importantly, the objective function is no longer differentiable and many derivativebased optimization algorithms cannot be applied to the problem $[9,10]$. The latter provides not accurate but reliable traffic emissions fitting functions that reflect the trends of emissions to some extent. On the other hand, the optimization models with explicit objective functions are much easier to solve. In this paper, we obtain the emissions fitting functions by collecting samples in MOVES, a kind of traffic emissions estimation software developed by EPA.

Let $K$ be the set of the air pollutants from mobile sources. For each pollutant $k \in K$, we define the pollutant amount released by a standard motor vehicle traveling one kilometer on link $a$ as emission factor $r_{a, k}$. Many empirical studies indicated that emission factor is associated with average speed [15]. Therefore, this paper adopts MOVES to fit the emission factor function $e_{a, k}\left(u_{a}\right)$ of the average speed $u_{a}$. Subsequently, the total emission of pollutant $k$ on link $a$ can be expressed by the following equation:

$$
r_{a, k}\left(v_{a}\right)=l_{a} \cdot v_{a} \cdot e_{a, k}\left(u_{a}\right) \quad \forall a \in \Omega^{r}, k \in K .
$$

Taking fitting the emission factor function of $\mathrm{NO}_{x}$ as an example, we input the meteorological data, source type, fuel type, and other deterministic parameters and then run MOVES with different average speed and get the corresponding emission factors. Finally, the emission factor function is fitted in OriginPro 8 and is shown as follows:

$$
e_{a}\left(u_{a}\right)=1.86022-0.03203 u_{a}+2.02256 u_{a}^{2} * 10^{-4} \text {. }
$$

3.2. Model Formulation. Robust optimization is an effective approach to address design problems with uncertainty. Specifically, the robust design focuses on the problem's worst-case scenario and aims at finding the solution that outperforms all other solutions in the worst-case scenario. Literature review shows that robust optimization approach has been applied to address many traffic problems. Chen et al. [29] developed a toll and capacity design model with demand uncertainty for a build operate-transfer roadway. Yin [30] proposed three robust signal timing design models to cope with traffic flow fluctuations. Later, these models were reformulated to determine the optimal road improvement scheme subject to demand uncertainty [31]. Chung et al. [32] adopted this method to optimize dynamic congestion pricing under demand uncertainty. See more recent reviews on robust network design or road pricing in Yin and Lou [33] and Koster et al. [34].

As a pioneering endeavor, this paper applies this method to design road speed limits against travel demand uncertainty. We suppose traffic managers are more concerned about the worst case rather than the best or average performance. Furthermore, it is assumed that the emissions from subway system are close to zero and the imposed speed limits are continuous variables.

For each O-D pair $w \in W$, the travel demand $q^{w}$ is assumed to be uncertain in this section. To model traffic demand uncertainty, we assume that the total demands belong to an uncertainty set denoted by $Q, q \in Q$. For a given demand vector $q \in Q, \Lambda_{q}$ denotes the corresponding feasible region of travel demand and flow distributions. This paper aims to find a speed limits scheme that minimizes the sum social costs of system travel time and vehicular emissions against the worst-case demand scenario. Thus, the robust speed limits design model can be formulated as a mathematical program with complementarity constraints (MPCC):

$$
\begin{array}{ll}
\min _{s} \max _{x, v, d, \pi} & \theta \sum_{a \in \Omega} \sum_{m \in M} \tilde{t}_{a, m}\left(v_{a, m}, s_{a, m}\right) v_{a, m} \\
& +\sum_{a \in \Omega^{r}} \sum_{k \in K} \rho_{k} \cdot e_{a, k}\left(u_{a}\right) v_{a} l_{a} \\
\text { s.t. } \quad & \text { Equation (11) } \\
& s_{a}^{\min } \leq s_{a} \leq s_{a}^{\max }, \quad \forall a \in \Omega^{r} \\
& (v, d) \in \Lambda_{q} \quad \forall q \in Q,
\end{array}
$$

where $u_{a}=l_{a} / \widetilde{t}_{a}, a \in \Omega^{r} . \rho_{k}$ represents the unit social cost of pollutant $k$ and consequently the objective is to minimize the total travel social cost. Equation (11) describes the bimodal network equilibrium conditions with complementarity constraints. $s_{a}^{\min }$ and $s_{a}^{\max }$ are, respectively, the lower bound and upper bound of $s_{a}$, In general, $s_{a}^{\max }$ is the free flow speed. $s_{a}^{\min }$ is associated with road conditions. In this paper, the demand uncertainty set $Q$ is expressed as an ellipsoid form [33]:

$$
Q=\left\{q \mid \sum_{w} \frac{\left(q^{w}-\mu^{w}\right)^{2}}{\left(\eta^{w}\right)^{2}} \leq \varepsilon^{2}\right\},
$$

where $\mu^{w}=(1 / 2)\left(q_{\min }^{w}+q_{\max }^{w}\right)$ and $\eta^{w}=(1 / 2)\left(q_{\max }^{w}-q_{\min }^{w}\right)$. Note that $q_{\max }^{w}$ and $q_{\min }^{w}$ are given. $\varepsilon$ implies the traffic managers' attitude toward risk. Generally, if traffic managers are risk averse, larger $\varepsilon$ would be appropriate, and vice versa [35].

Since that the feasible region is nonconvex and standard stationarity conditions may not hold [36], MPCC is very difficult to solve.

\section{Solution Algorithms}

The proposed robust speed limit design model can be solved by conducting a cutting-plane scheme, whose basic idea is to generate one extreme point of the feasible region at a time, each of which produces a constraint that cuts away part of the region not feasible to the original problem [37]. When applied to the proposed model in this paper, the specific procedures are listed as follows.

Step 1. Create a subset $Q^{\prime}$ of demand set $Q$. Set $N=1$, select a demand vector $q^{1} \in Q$, and let $Q^{\prime}$ be $\left\{q^{1}\right\}$. Initialize the speed limits scheme $s^{0}$ and solve bimodal UE model in Section 2 to obtain a feasible solution $(x, v, \pi, d)$ and go to Step 2 .

Step 2. Let $\Delta^{n}=\theta \sum_{a \in \Omega} \sum_{m \in M} \widetilde{t}_{a, m}^{n}\left(v_{a, m}^{n}, s_{a, m}\right) v_{a, m}^{n}+\sum_{a \in \Omega^{r}}$ $\sum_{k \in K} \rho_{k} \cdot e_{a}^{n}\left(u_{a}^{n}\right) v_{a}^{n} l_{a}, n \in\{1,2, \ldots, N\}$. Introduce a new variable $\psi$, so the min-max model can be reformulated as 
Subproblem 1. Solve it to find the optimal speed limits $s^{N *}$ and $\psi^{N *}$ within the subset $Q^{\prime}$.

\section{Subproblem 1. We have}

$$
\begin{aligned}
& \min _{x, v, \pi, s, \Delta} \psi \\
& \text { s.t. Equation (16) } \\
& x_{a, m}^{w, n}\left(\widetilde{t}_{a, m}^{n}\left(v_{a, m}^{n}, s_{a, m}\right)-\pi_{i, m}^{w, n}+\pi_{j, m}^{w, n}\right)=0, \\
& \forall a=(i, j) \in \Omega, w \in W, \\
& m \in M, n \in\{1,2, \ldots, N\} \\
& \widetilde{t}_{a, m}^{n}\left(v_{a, m}^{n}, s_{a, m}\right)-\pi_{i, m}^{w, n}+\pi_{j, m}^{w, n} \geq 0, \\
& \forall a=(i, j) \in \Omega, w \in W, \\
& m \in M, n \in\{1,2, \ldots, N\} \\
& d_{m}^{w, n}=q^{w, n} \frac{\exp \left(-\theta\left(\pi_{o, m}^{w, n}-\pi_{d, m}^{w, n}\right)-\tau_{m}^{w}\right)}{\sum_{m \in M} \exp \left(-\theta\left(\pi_{o, m}^{w, n}-\pi_{d, m}^{w, n}\right)-\tau_{m}^{w}\right)}, \\
& \forall w \in W, q^{n} \in Q^{\prime}, n \in\{1,2, \ldots, N\} \\
& \Delta^{n} \leq \psi \quad n \in\{1,2, \ldots, N\} \\
& \left(v^{n}, d^{n}\right) \in \Lambda_{q^{n}} \quad q^{n} \in Q^{\prime}, n \in\{1,2, \ldots, N\} .
\end{aligned}
$$

This above model has complementarity constraints and can be solved by manifold suboptimization algorithm developed by Lawphongpanich and Yin [38]. The basic idea of the algorithm is to solve a sequence of restricted nonlinear optimization problems to obtain a strongly stationary solution.

Step 3. Let $\phi=\theta \sum_{a \in \Omega} \sum_{m \in M} \widetilde{t}_{a, m}\left(v_{a, m}, s_{a, m}\right) v_{a, m}+$ $\sum_{a \in \Omega^{r}} \sum_{k \in K} \rho_{k} \cdot e_{a, k}\left(u_{a}\right) v_{a} l_{a}$. Set the solution of Subproblem 1 as the initial value and solve the following Subproblem 2 to find the worst-case demand scenario $q^{N *}$ in the set $Q$ that maximizes the objective function whose optimal value is noted as $\phi^{N *}$.

Subproblem 2. We have

$$
\begin{array}{ll}
\max _{x, v, d, \pi} & \phi \\
\text { s.t. } & \text { Equations }(11),(17) .
\end{array}
$$

As formulated, this above model is MPCC and can be also solved by manifold suboptimization algorithm developed by Lawphongpanich and Yin [38].

Step 4. If $\phi^{N *} \leq \psi^{N *}$, then $s^{N *}$ is the best solution. Otherwise, $Q^{\prime}=Q^{\prime} \cup q^{N *}$ and $N=N+1$. Go back to Step 2, and set the solution of Subproblem 2 as the initial value of Subproblem 1 .
TABLE 1: The parameters of road links.

\begin{tabular}{cccc}
\hline Link & $t_{0}(\min )$ & Capacity & Length $(\mathrm{km})$ \\
\hline$(1,2)$ & 6 & 8000 & 8 \\
$(1,4)$ & 5 & 5000 & 7 \\
$(2,3)$ & 2 & 3000 & 3 \\
$(2,5)$ & 3 & 3500 & 4 \\
$(3,6)$ & 2 & 3300 & 3 \\
$(4,2)$ & 1 & 8000 & 1.5 \\
$(4,5)$ & 4 & 8000 & 6 \\
$(5,6)$ & 3.5 & 8000 & 5 \\
\hline
\end{tabular}

In general, we can figure out the robust speed limit by solving Subproblem 1 with respect to a subset $Q^{\prime} \subset Q$ that contains $N$ demand vectors and solving Subproblem 2 to generate new demand vectors to expand $Q^{\prime}$. Repeat this procedure until the output meets some stopping criterion.

\section{Numerical Examples}

This section illustrates the proposed model and algorithm with a virtual bimodal network shown in Figure 4 that comprises two O-D pairs: $1-5$ and $4-6$, respectively. Note that the uncertain total demand vector $q$ varies within the specified set $Q$ by (14), where $q_{\max }=[10000,12000]^{T}, q_{\min }=$ $[3000,4000]^{T}$, and $\varepsilon=1.2$ in this example. To simplify the demonstration, we only set speed limits on links $(1,4)$ and $(5,6)$. The lower bound of each speed limit variable is $40 \mathrm{~km} / \mathrm{h}$, and the upper bound is the free flow speed. Our goal is to find the optimal speed limit scheme within the specified range.

The parameters of road links are displayed in Table 1. We use the BPR function (US Bureau of Public Roads), that is, $t\left(v_{a}\right)=t_{a}^{0}\left(1+0.15\left(v_{a} / C_{a}\right)^{4}\right)$, to calculate the travel time on road links without speed limits.

As shown in Figure 3, the subway network is constituted with 6 stations and 3 lines. Note that S2 (2a), S3 (3a), and S4 (4a) are transfer stations. The parameters of subway links, embarking and alighting links, and transfer links are displayed in Table 2. We can see that the waiting time on alighting links is 0 , consistent with common sense. In addition, we assume the following walking time function has the similar form as BPR function, where $\alpha=0.15$ and $\beta=2$.

$$
\operatorname{TP}\left(v_{a}\right)=t_{a}^{0}\left(1+\alpha\left(\frac{v_{a}}{C_{a}}\right)^{\beta}\right) .
$$

Let $\tau_{r}^{1-5}=\tau_{r}^{4-6}=5 \$$, and let $\tau_{s}^{1-5}=\tau_{s}^{4-6}=2 \$$. To simplify the calculation, we only consider the $\mathrm{NO}_{x}$ emissions that can be figured out by (13) and (14). Because the unit social cost of $\mathrm{NO}_{x}$ is much higher than travel time, we set $\theta=0.5$ and $\rho=3$ in this example. Through applying the solution algorithm proposed in the previous section, we get the optimum displayed in Tables 3 and 4.

In Table 3, it is obvious to see that under the worst-case demand scenario, the sharing rate of private cars in this network is $69.94 \%$, higher than the sharing rate of subway. 
TABLE 2: The parameters of links in subway system.

\begin{tabular}{|c|c|c|c|c|c|}
\hline & Link & $\mathrm{TR}+\mathrm{TS}(\mathrm{min})$ & $\mathrm{TW}(\min )$ & $t_{0}(\min )$ & Capacity \\
\hline \multirow{3}{*}{ Subway links on orange line } & $(\mathrm{S} 1, \mathrm{~S} 2)$ & 4 & - & - & 10000 \\
\hline & $(\mathrm{S} 2, \mathrm{~S} 4)$ & 3.5 & - & - & 10000 \\
\hline & $(\mathrm{S} 4, \mathrm{~S} 6)$ & 2 & - & - & 10000 \\
\hline \multirow{2}{*}{ Subway links on green line } & $(2 \mathrm{a}, 3 \mathrm{a})$ & 6 & - & - & 10000 \\
\hline & $(3 a, 4 a)$ & 3 & - & - & 10000 \\
\hline \multirow{3}{*}{ Subway links on purple line } & $(\mathrm{S} 1, \mathrm{~S} 3)$ & 3 & - & - & 10000 \\
\hline & $(\mathrm{S} 3, \mathrm{~S} 5)$ & 3.5 & - & - & 10000 \\
\hline & $(\mathrm{S} 5, \mathrm{S6})$ & 4 & - & - & 10000 \\
\hline \multirow{3}{*}{ Embarking links } & $(1, \mathrm{~S} 1)$ & - & 1 & 3 & 10000 \\
\hline & $(4, S 2)$ & - & 1 & 3 & 10000 \\
\hline & $(4,2 \mathrm{a})$ & - & 1 & 3 & 10000 \\
\hline \multirow{3}{*}{ Alighting links } & $(S 4,5)$ & - & 0 & 3.5 & 10000 \\
\hline & $(4 a, 5)$ & - & 0 & 3.5 & 10000 \\
\hline & $(\mathrm{S} 6,6)$ & - & 0 & 3.5 & 10000 \\
\hline \multirow{4}{*}{ Transfer links } & $(\mathrm{S} 2,2 \mathrm{a})$ & - & 1 & 2 & 10000 \\
\hline & $(\mathrm{S} 3,3 \mathrm{a})$ & - & 1 & 2.5 & 10000 \\
\hline & $(3 a, S 3)$ & - & 1 & 2 & 10000 \\
\hline & $(4 a, S 4)$ & - & 1 & 2 & 10000 \\
\hline
\end{tabular}

TABLE 3: The worst-case demand and allocation results.

\begin{tabular}{lccc}
\hline O-D pairs & Worst-case demand & Demand on private cars & Demand on subway \\
\hline $1-5$ & 8519.519 & 6443.683 & 2075.837 \\
$4-6$ & 10488.581 & 6851.332 & 3637.249 \\
\hline
\end{tabular}

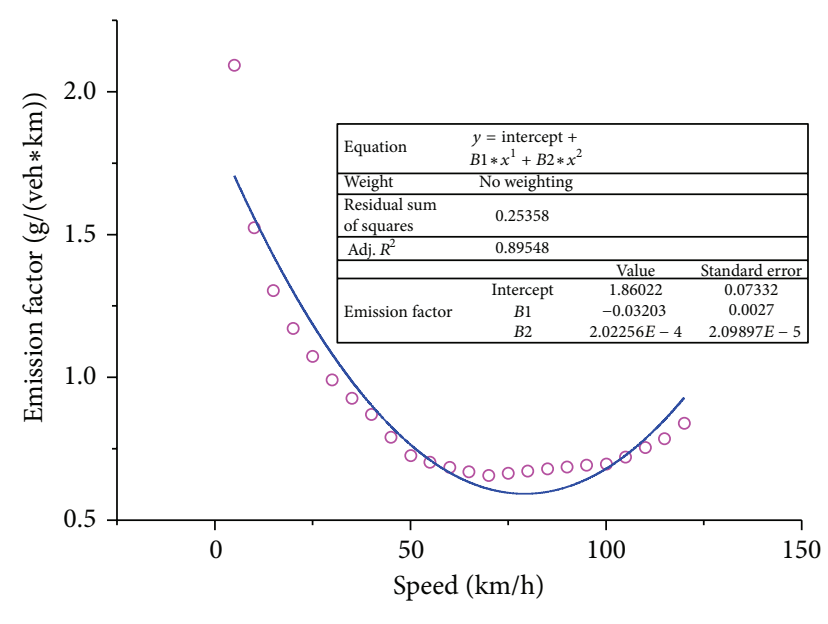

- $\mathrm{NO}_{X}$ emission factor

_ Polynomial fitting result

FIGURE 3: Emission factor fitting function of $\mathrm{NO}_{x}$.

In the light of multinomial Logit model, the sharing rate of each transport mode is associated with the minimum O-D travel time, parking fare, and ticket fare. Therefore, different fare setting would impact the modal split result.

The optimal road speed limits settings are presented in Table 4. It can be found that the best imposed speed limit on Link $(1,4)$ reaches the upper bound, but on Link

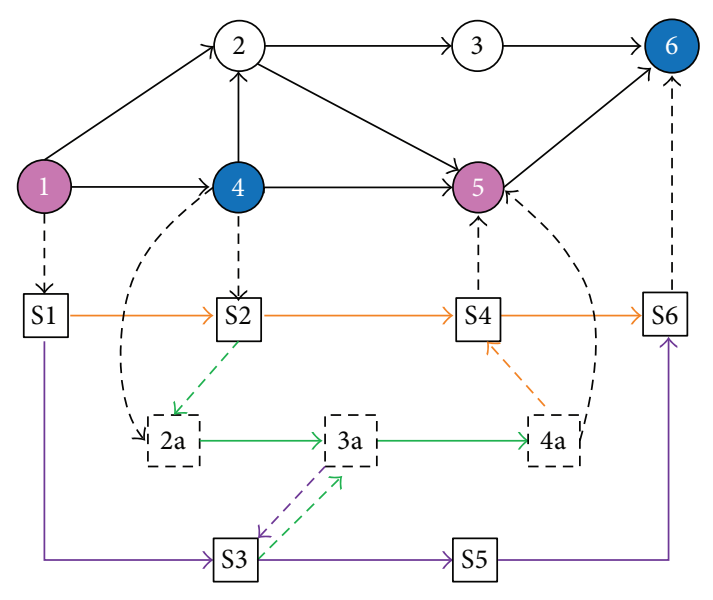

FIGURE 4: A hypothetical bimodal network.

$(5,6)$, the opposite situation happens. This interesting result indicates that imposing the same or similar speed limits on road links in practical traffic management could not benefit traffic congestion or emissions reduction, even worsen them sometimes. Other link-specific results, that is, link flow distributions, average travel time, and emission factors, are as well listed in Table 4.

Now we conduct a sensitive analysis on parking fare. Specifically, except the parking fare between each O-D pair, all the other parameters are fixed as the above mentioned. 
TABLE 4: The link-specific results.

\begin{tabular}{|c|c|c|c|c|c|c|c|}
\hline Road links & $\begin{array}{l}\text { Link flow } \\
\text { (veh) }\end{array}$ & $\begin{array}{l}\text { Travel time } \\
(\mathrm{min})\end{array}$ & $\begin{array}{c}\text { Emission factor } \\
(\mathrm{g} / \mathrm{km})\end{array}$ & $\begin{array}{l}\text { Speed limits } \\
(\mathrm{km} / \mathrm{h})\end{array}$ & $\begin{array}{c}\text { Links in subway } \\
\text { system }\end{array}$ & $\begin{array}{l}\text { Link flow } \\
\text { (passenger) }\end{array}$ & $\begin{array}{c}\text { Travel time } \\
(\mathrm{min})\end{array}$ \\
\hline$(1,2)$ & 3001.517 & 6.018 & 0.592 & & $(\mathrm{~S} 1, \mathrm{~S} 2)$ & 1176.966 & 4 \\
\hline$(1,4)$ & 3442.166 & 5.168 & 0.593 & 84 & $(\mathrm{~S} 2, \mathrm{~S} 4)$ & 898.871 & 3.5 \\
\hline$(2,3)$ & 5277.154 & 4.872 & 0.953 & & $(\mathrm{~S} 4, \mathrm{~S} 6)$ & 0.210 & 2 \\
\hline$(2,5)$ & 3001.517 & 3.243 & 0.598 & & $(2 a, 3 a)$ & 4814.005 & 6 \\
\hline$(3,6)$ & 5277.154 & 3.962 & 0.822 & & $(3 a, 4 a)$ & 898.999 & 3 \\
\hline$(4,2)$ & 5277.154 & 1.028 & 0.606 & & $(\mathrm{~S} 1, \mathrm{~S} 3)$ & 0.082 & 3 \\
\hline$(4,5)$ & 5016.344 & 4.093 & 0.608 & & $(\mathrm{~S} 3, \mathrm{~S} 5)$ & 3637.167 & 3.5 \\
\hline \multirow[t]{11}{*}{$(5,6)$} & 1574.178 & 5.770 & 0.742 & 52 & $(\mathrm{~S} 5, \mathrm{~S} 6)$ & 0.082 & 4 \\
\hline & & & & & $(1, \mathrm{~S} 1)$ & 2075.837 & 4.019 \\
\hline & & & & & $(4, \mathrm{~S} 2)$ & 3637.104 & 4.060 \\
\hline & & & & & $(4,2 a)$ & 0.145 & 4.000 \\
\hline & & & & & $(S 4,5)$ & 1176.932 & 3.507 \\
\hline & & & & & $(4 a, 5)$ & 898.904 & 3.504 \\
\hline & & & & & $(\mathrm{S} 6,6)$ & 3637.249 & 3.569 \\
\hline & & & & & $(\mathrm{S} 2,2 \mathrm{a})$ & 0.065 & 3.000 \\
\hline & & & & & $(\mathrm{S} 3,3 \mathrm{a})$ & 898.887 & 3.503 \\
\hline & & & & & $(3 a, S 3)$ & 0.098 & 3.000 \\
\hline & & & & & $(4 a, S 4)$ & 0.095 & 3.000 \\
\hline
\end{tabular}

TABLE 5: The optimal speed limit schemes under 81 scenarios.

\begin{tabular}{lccccccccc}
\hline & 2 & 3 & 4 & 5 & 6 & 7 & 8 & 9 \\
\hline 2 & $(84,86)$ & $(42,40)$ & $(84,42)$ & $(40,86)$ & $(84,59)$ & $(84,40)$ & $(84,86)$ & $(84,63)$ & $(84,60)$ \\
3 & $(44,40)$ & $(50,42)$ & $(44,40)$ & $(56,85)$ & $(84,86)$ & $(56,85)$ & $(56,86)$ & $(84,86)$ & $(84,60)$ \\
4 & $(63,42)$ & $(84,86)$ & $(84,86)$ & $(62,52)$ & $(84,70)$ & $(60,86)$ & $(84,86)$ & $(63,85)$ & $(84,72)$ \\
5 & $(71,42)$ & $(84,86)$ & $(84,42)$ & $(84,52)$ & $(59,57)$ & $(71,85)$ & $(71,86)$ & $(71,86)$ & $(84,86)$ \\
6 & $(74,42)$ & $(84,86)$ & $(74,42)$ & $(84,86)$ & $(84,86)$ & $(84,86)$ & $(62,46)$ & $(84,40)$ & $(84,86)$ \\
7 & $(84,86)$ & $(83,40)$ & $(81,86)$ & $(82,52)$ & $(84,70)$ & $(84,84)$ & $(84,86)$ & $(68,83)$ & $(84,84)$ \\
8 & $(84,40)$ & $(84,40)$ & $(84,40)$ & $(84,86)$ & $(84,60)$ & $(84,86)$ & $(84,86)$ & $(72,62)$ & $(67,61)$ \\
9 & $(84,40)$ & $(84,40)$ & $(84,40)$ & $(84,52)$ & $(84,55)$ & $(40,86)$ & $(47,40)$ & $(84,62)$ & $(79,47)$ \\
10 & $(84,40)$ & $(84,40)$ & $(84,40)$ & $(80,40)$ & $(84,70)$ & $(84,75)$ & $(84,86)$ & $(84,61)$ & $(84,86)$ \\
\hline
\end{tabular}

Starting from $2 \$$, we gradually increase the parking fare between each O-D pair by $1 \$$, until both of them attain $10 \$$. Thus, the total number of parking fare scenarios is 81 . By using the proposed model and solution algorithm, we obtain the objective value, modal split, and optimal speed limits scheme with respect to each parking fare scenario.

Observing the change trends of the following objective values under 81 scenarios as shown in Figure 5, we intuitively conclude that higher parking fares result in lower transportation social cost. However, this conclusion does not always hold when one parking fare is fixed, such that the other parking fares vary from $2 \$$ to $10 \$$. For example, $g[2,3]=$ $521527.486>g[2,2]=472145.734$, where $g$ represents the objective value and $\left[\tau_{r}^{1-5}, \tau_{r}^{4-6}\right]$ denotes the parking fare scenario. After analysis, the reasons are considered as follows. (i) The obtained worst-case demand for each parking fare scenario is independent and different, given that the total travel demand is subjected to an uncertain set. (ii) The solution and best objective value for our robust speed limit design model is local optimum instead of global optimum. (iii) Initialization probably impacts the optimal objective value.

Figure 6 demonstrates the modal split rate of private cars under 81 parking fare scenarios. Overall, it is straightforward that private cars carry a large proportion of traffic demand in case of cutting parking fares. On the contrary, along with rising parking fares, the traffic demand proportion loaded by private cars would decrease. However, we can observe that this lemma is violated under some parking fare scenarios as shown in Figure 6. This is because the worst-case total travel demand is changeable. It cannot be guaranteed that the drop rate of total demand carried by private cars is great than that of worst-case total travel demand with higher parking fares.

Table 5 displays the optimal speed limit schemes under 81 parking fare scenarios. It is easily found that the optimal 


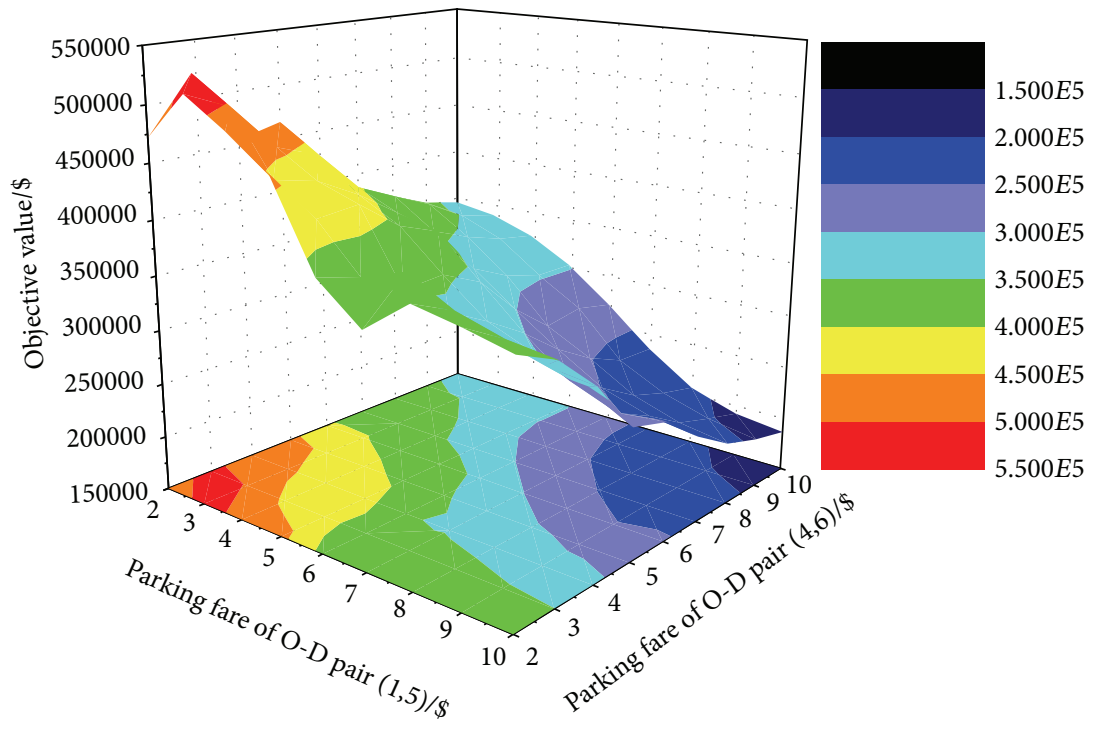

Figure 5: The objective values under 81 scenarios.

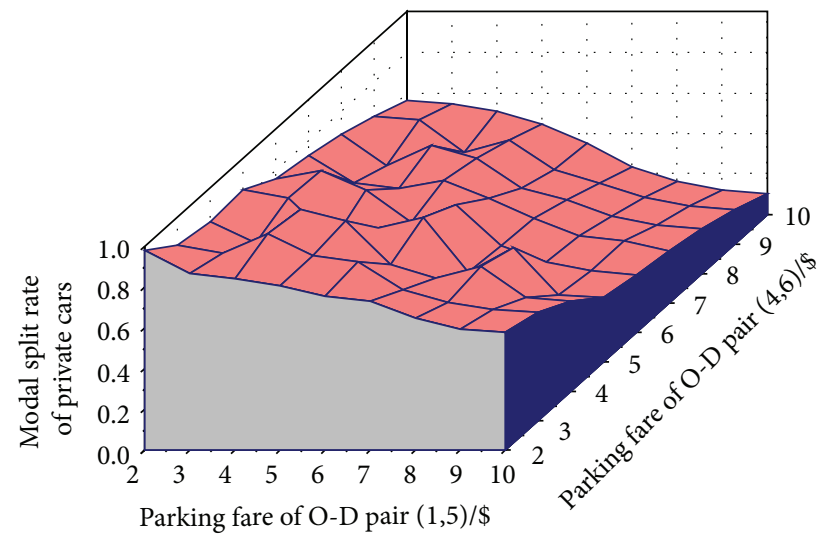

FIGURE 6: Modal split rate of private cars under 81 scenarios.

speed limit schemes with different parking fares are quite untraceable. Therefore, parking fares significantly impact the optimal imposed speed limits, and an integrated design of speed limits and parking fares is more recommended.

Similarly, different subway fare scenarios would have an influence on the best objective value, modal split pattern, and optimal speed limit scheme. We do not repeatedly conduct the sensitivity analysis of subway fare in this paper. Still, it can be concluded that an integrated design of speed limits and extra fares, that is, parking fares and subway fares, is more recommended.

\section{Conclusions}

This paper investigated the design of robust speed limits scheme in an attempt to reduce transportation externalities for a bimodal transportation system including private cars and subway. First of all, a synthesized travel time function under speed limits was established for such a system. The bimodal network equilibrium model with speed limits was developed to provide a feasible initial solution for the subsequent robust speed limits scheme design model. Assuming that the total demand vector belongs to a given demand set, we formulated the robust speed limits scheme design problem as a min-max MPCC model. To estimate traffic emissions more reliably, a professional emissions calculation tool, MOVES, was employed to simulate emission factor functions. Then we utilize the cutting plane method framework to solve the proposed model. At the end, a numerical example was demonstrated to verify the proposed model and solution algorithm. Besides, we conducted a sensitivity analysis of parking fares in the example. The results showed that the objective value and modal split rate of private cars did not necessarily decline when increasing the parking fares, which contradicted with our intuitions. Consequently, our further study should design the speed limits and extra fares, that is, parking fares and subway fares, in an integrated manner.

\section{Conflict of Interests}

The authors declare that there is no conflict of interests regarding the publication of this paper.

\section{Acknowledgments}

The research is mainly supported by grants from the Research Project (2012BAJ05B04) of national science and technology support program of China (Project no. 2012BAJ05B00) and Beijing higher education young elite teacher project. 


\section{References}

[1] H. Yang and H. J. Huang, Mathematical and Economic Theory of Road Pricing, Elsevier, 2005.

[2] T. Tsekeris and S. Voss, "Design and evaluation of road pricing: state-of-the-art and methodological advances," Netnomics, vol. 10, no. 1, pp. 5-52, 2009.

[3] A. de Palma and R. Lindsey, "Traffic congestion pricing methodologies and technologies," Transportation Research Part C, vol. 19, no. 6, pp. 1377-1399, 2011.

[4] A. C. Pigou, The Economics of Welfare, Macmillan, London, UK, 1920.

[5] H. C. Joksch, "An empirical relation between fatal accident involvement per accident involvement and speed," Accident Analysis \& Prevention, vol. 7, no. 2, pp. 129-132, 1975.

[6] X. Qu, Q. Meng, and Z. Liu, "Estimation of number of fatalities caused by toxic gases due to fire in road tunnels," Accident Analysis and Prevention, vol. 50, pp. 616-621, 2013.

[7] TxDOT Online Manuals, 2013, http://onlinemanuals.txdot.gov/ manuals/index.html.

[8] M. Madireddy, B. de Coensel, A. Can et al., "Assessment of the impact of speed limit reduction and traffic signal coordination on vehicle emissions using an integrated approach," Transportation Research D, vol. 16, no. 7, pp. 504-508, 2011.

[9] Y. Yang, H. Lu, Y. Yin, and H. Yang, "Optimization of variable speed limits for efficient, safe, and sustainable mobility," Transportation Research Record, no. 2333, pp. 37-45, 2013.

[10] Y. Yang, Y. Yin, and H. Lu, "Designing emission charging schemes for transportation conformity," Journal of Advanced Transportation, 2013.

[11] J. E. Woolley, C. B. Dyson, and M. A. P. Taylor, "Impacts of lower speed limits in South Australia," IATSS Research, vol. 26, no. 2, pp. 6-17, 2002.

[12] H. Yang, X. Wang, and Y. Yin, "The impact of speed limits on traffic equilibrium and system performance in networks," Transportation Research B, vol. 46, no. 10, pp. 1295-1307, 2012.

[13] S. Wang, "Efficiency and equity of speed limits in transportation networks," Transportation Research C, vol. 32, pp. 61-75, 2013.

[14] Y. Yin and S. Lawphongpanich, "Internalizing emission externality on road networks," Transportation Research D: Transport and Environment, vol. 11, no. 4, pp. 292-301, 2006.

[15] L. Yu, S. Jia, and Q. Shi, "Research on transportation-related emissions: Current status and future directions," Journal of the Air and Waste Management Association, vol. 59, no. 2, pp. 183195, 2009.

[16] M. Abdulaal and L. J. Leblanc, "Methods for combining modal split and equilibrium assignment models," Transportation Science, vol. 13, no. 4, pp. 292-314, 1979.

[17] S. Dafermos, "The general multimodal network equilibrium problem with elastic demand," Networks, vol. 12, no. 1, pp. 57-72, 1982.

[18] R. García and A. Marín, "Network equilibrium with combined modes: models and solution algorithms," Transportation Research B, vol. 39, no. 3, pp. 223-254, 2005.

[19] X. Fu and W. H. K. Lam, "A network equilibrium approach for modelling activity-travel pattern scheduling problems in multimodal transit networks with uncertainty," Transportation, vol. 41, no. 1, pp. 37-55, 2014.
[20] J. Q. Ying and H. Yang, "Sensitivity analysis of stochastic user equilibrium flows in a bi-modal network with application to optimal pricing," Transportation Research B, vol. 39, no. 9, pp. 769-795, 2005

[21] Y. Hamdouch, M. Florian, D. W. Hearn, and S. Lawphongpanich, "Congestion pricing for multi-modal transportation systems," Transportation Research B: Methodological, vol. 41, no. 3, pp. 275-291, 2007.

[22] D. Wu, Y. Yin, and S. Lawphongpanich, "Pareto-improving congestion pricing on multimodal transportation networks," European Journal of Operational Research, vol. 210, no. 3, pp. 660-669, 2011.

[23] W. H. K. Lam, Z. Y. Gao, K. S. Chan, and H. Yang, "A stochastic user equilibrium assignment model for congested transit networks," Transportation Research B, vol. 33, no. 5, pp. 351-368, 1999.

[24] B. F. Si, J. C. Long, and Z. Y. Gao, “Optimization model and algorithm for mixed traffic of urban road network with flow interference," Science in China. Series E, vol. 51, no. 12, pp. 2223 2232,2008

[25] M. S. Bazaraa, H. D. Sherali, and C. M. Shetty, Nonlinear Programming: Theory and Algorithms, John Wiley \& Sons, 3rd edition, 2006.

[26] R. K. Ahuja, T. L. Magnanti, and J. B. Orlin, Network Flows: Theory, Algorithms, and Applications, Prentice Hall, Englewood Cliffs, NJ, USA, 1993.

[27] S. Lawphongpanich and Y. Yin, "Nonlinear pricing on transportation networks," Transportation Research Part C, vol. 20, no. 1, pp. 218-235, 2012.

[28] L. S. Matott, B. A. Tolson, and M. Asadzadeh, "A benchmarking framework for simulation-based optimization of environmental models," Environmental Modelling and Software, vol. 35, pp. 19-30, 2012.

[29] A. Chen, K. Subprasom, and Z. Ji, "Mean-variance model for the build-operate transfer scheme under demand uncertainty," Transportation Research Record, no. 1857, pp. 93-101, 2003.

[30] Y. Yin, "Robust optimal traffic signal timing," Transportation Research B, vol. 42, no. 10, pp. 911-924, 2008.

[31] Y. Yin, S. M. Madanat, and X. Lu, "Robust improvement schemes for road networks under demand uncertainty," European Journal of Operational Research, vol. 198, no. 2, pp. 470479, 2009.

[32] B. D. Chung, T. Yao, T. L. Friesz, and H. Liu, "Dynamic congestion pricing with demand uncertainty: a robust optimization approach," Transportation Research Part B, vol. 46, no. 10, pp. 1504-1518, 2012.

[33] Y. Yin and Y. Lou, "Robust optimization approach for transportation network design under demand uncertainty," in ICCTP: Critical Issues in Transportation Systems Planning, Development, and Management, 2009.

[34] A. M. C. Koster, M. Kutschka, and C. Raack, "Robust network design: formulations, valid inequalities, and computations," Networks, vol. 61, no. 2, pp. 128-149, 2013.

[35] A. Ben-Tal and A. Nemirovski, "Robust optimization-methodology and applications," Mathematical Programming B, vol. 92 , no. 3, pp. 453-480, 2002. 
[36] H. Scheel and S. Scholtes, "Mathematical programs with complementarity constraints: stationarity, optimality, and sensitivity," Mathematics of Operations Research, vol. 25, no. 1, pp. 1-22, 2000.

[37] S. Lawphongpanich and D. W. Hearn, "An MPEC approach to second-best toll pricing," Mathematical Programming A: Publication of the Mathematical Programming Society, vol. 101, no. 1, pp. 33-55, 2004.

[38] S. Lawphongpanich and Y. Yin, "Solving the Pareto-improving toll problem via manifold suboptimization," Transportation Research C: Emerging Technologies, vol. 18, no. 2, pp. 234-246, 2010. 


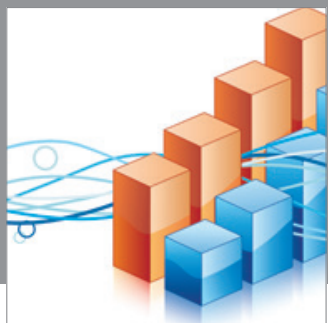

Advances in

Operations Research

mansans

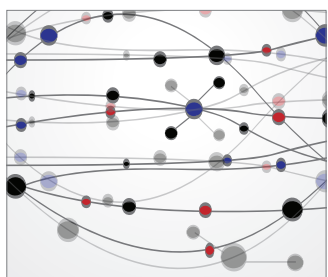

The Scientific World Journal
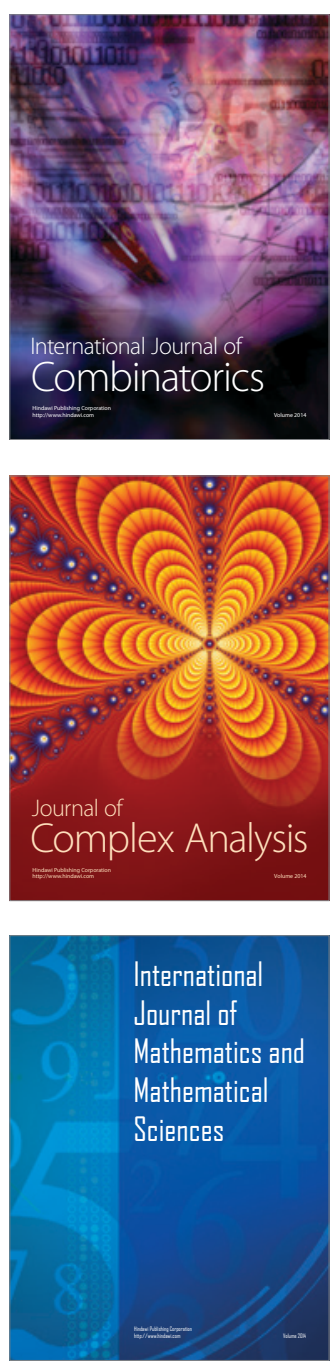
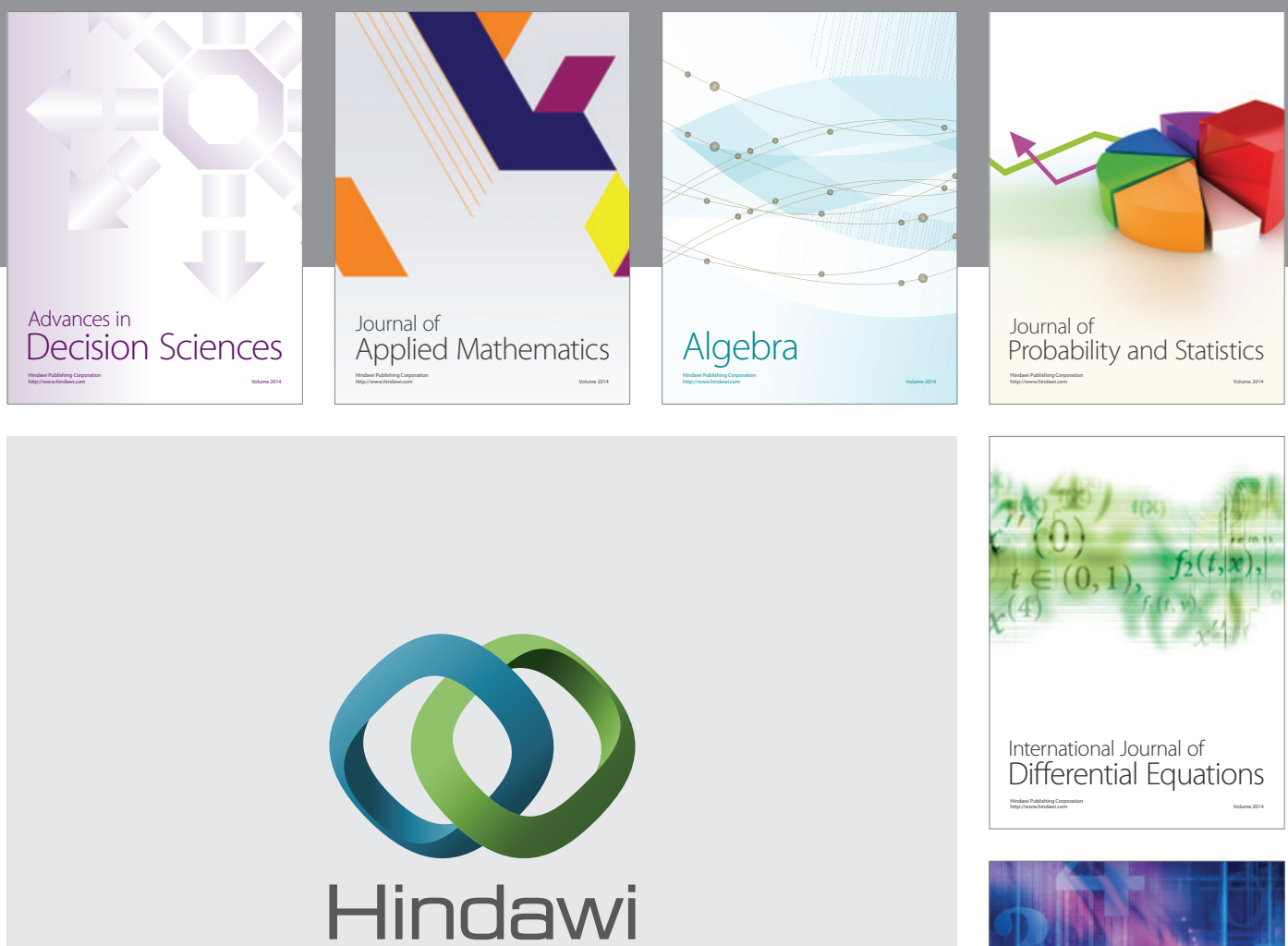

Submit your manuscripts at http://www.hindawi.com
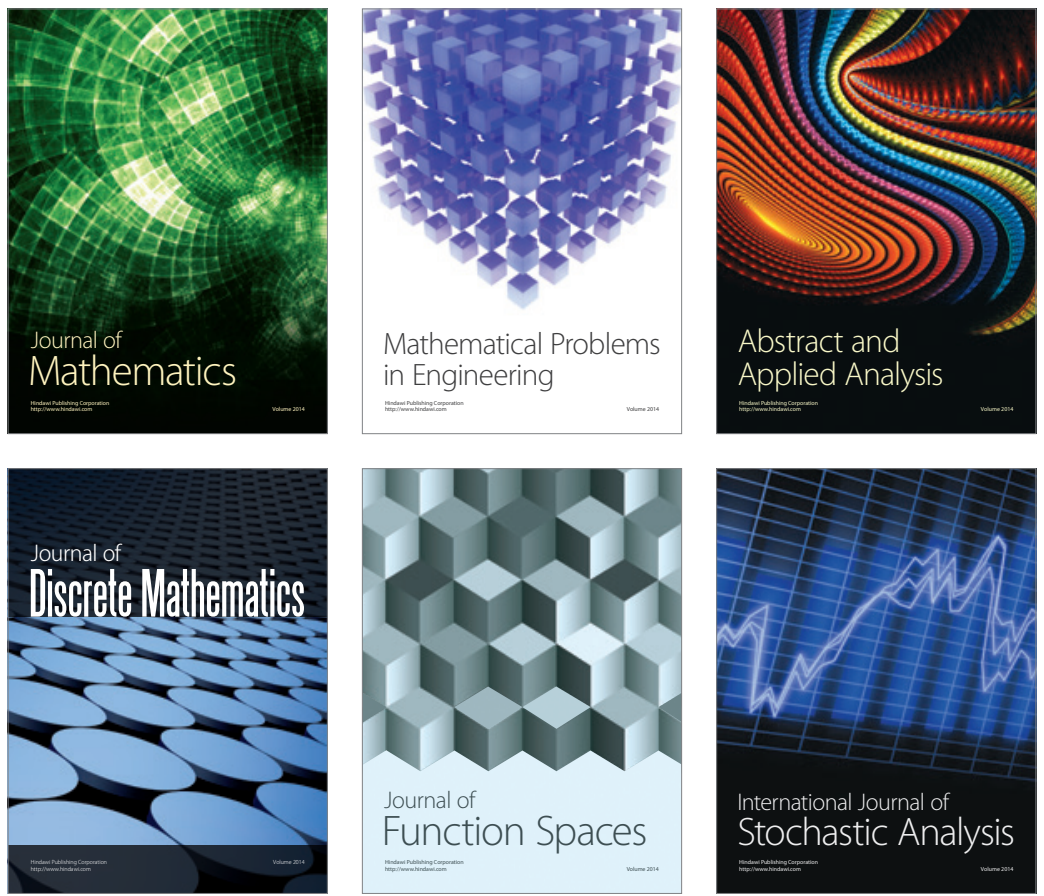

Journal of

Function Spaces

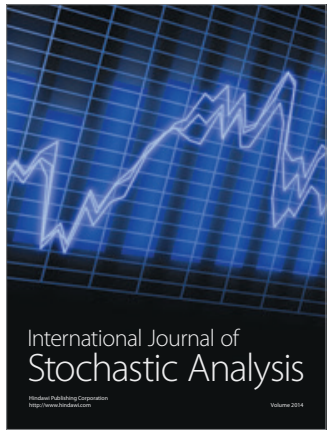

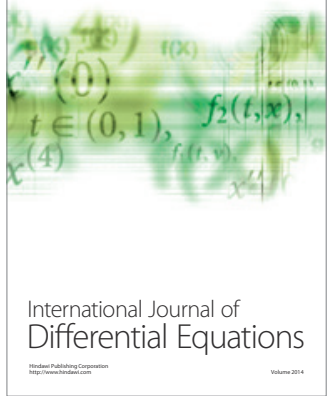
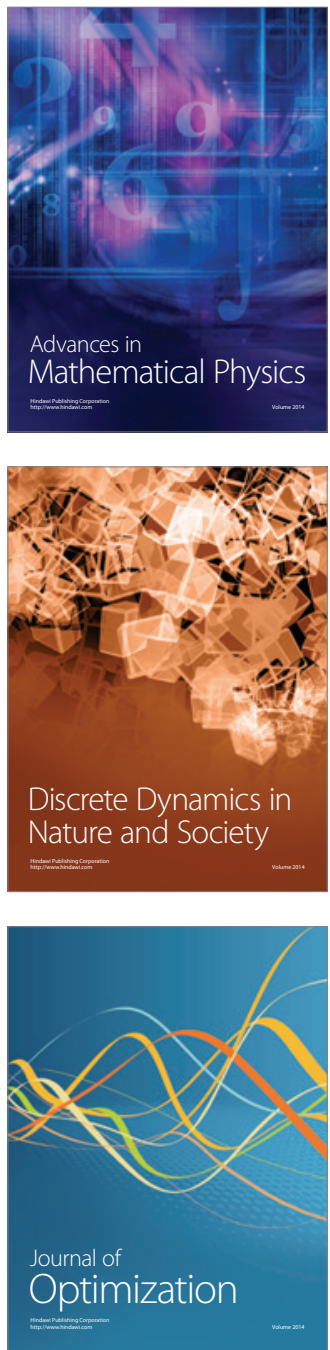